Bull. Korean Math. Soc. 40 (2003), No. 3, pp. 489-501

\title{
OSCILLATION OF SECOND ORDER NONLINEAR DELAY DIFFERENCE EQUATIONS
}

\author{
S. H. SAKER
}

ABstraCT. In this paper we shall consider the nonlinear delay difference equation

$$
\Delta\left(p_{n} \Delta x_{n}\right)+q_{n} f\left(x_{n-\sigma}\right)=0, \quad n=0,1,2, \ldots
$$

when $\sum_{n=n_{0}}^{\infty} \frac{1}{p_{n}}<\infty$. We will establish some sufficient conditions which guarantee that every solution is oscillatory or converges to zero.

\section{Introduction}

Recently, there has been an increasing interest in the study of oscillation and asymptotic behavior of solutions of second order difference equations, see, e.g., [1]-[16], [18]-[25]. Following this trend, in this paper we shall consider the nonlinear delay difference equation

$$
\Delta\left(p_{n} \Delta x_{n}\right)+q_{n} f\left(x_{n-\sigma}\right)=0, \quad n=0,1,2, \ldots,
$$

where $\Delta$ denotes the forward difference operator $\Delta x_{n}=x_{n+1}-x_{n}$ for any sequence $\left\{x_{n}\right\}$ of real numbers, $\sigma$ is nonnegative integer, $\left\{p_{n}\right\}_{n=0}^{\infty}$ and $\left\{q_{n}\right\}_{n=0}^{\infty}$ are sequences of real numbers such that $p_{n}>0, q_{n} \geq 0$ and $\left\{q_{n}\right\}$ has a positive subsequence, and $f$ is a continuous, nondecreasing real valued function which satisfies

$$
u f(u)>0 \text { for } u \neq 0 \text { and } f(u) / u \geq \gamma>0 .
$$

By a solution of (1.1) we mean a nontrivial sequence $\left\{x_{n}\right\}$ which is defined for $n \geq-\sigma$ and satisfies equation (1.1) for $n=0,1,2 \ldots$. Clearly if

$$
x_{n}=A_{n} \quad \text { for } \quad n=-\sigma, \ldots,-1,0,
$$

Received April 1, 2002.

2000 Mathematics Subject Classification: 39A10.

Key words and phrases: oscillation, nonlinear delay difference equations. 
are given, then equation (1.1) has a unique solution satisfying the initial conditions (1.3). A solution $\left\{x_{n}\right\}$ of (1.1) is said to be oscillatory if for every $n_{1}>0$ there exists an $n \geq n_{1}$ such that $x_{n} x_{n+1} \leq 0$, otherwise it is nonoscillatory. Equation (1.1) is said to be oscillatory if all its solutions are oscillatory.

In most of the above mentioned papers, the authors considered the linear or nonlinear difference equations and gave some sufficient conditions for oscillation when $p_{n}>0$, and

$$
\sum_{n=0}^{\infty} \frac{1}{p_{n}}=\infty
$$

In [21], Zhang, considered the equation

$$
\Delta\left(p_{n} \Delta x_{n}\right)+q_{n} x_{n+1}^{\gamma}=0, \quad n=0,1,2, \ldots,
$$

when

$$
\sum_{n=0}^{\infty} \frac{1}{p_{n}}<\infty
$$

and proved the following: Every solution of superlinear equation (1.5) oscillates if and only if

$$
\sum_{n=n_{0}}^{\infty} q_{n}\left(\rho_{n+1}\right)^{\gamma}=\infty, \quad \text { where } \rho_{n}=\sum_{i=n}^{\infty} \frac{1}{p_{i}}, \quad \gamma>1,
$$

and every solution of sublinear equation (1.5) oscillates if and only if

$$
\sum_{n=n_{0}}^{\infty} q_{n}\left(\rho_{n+1}\right)=\infty \quad \text { where } \rho_{n}=\sum_{i=n}^{\infty} \frac{1}{p_{i}}, \quad 0<\gamma<1 .
$$

In [4], Arul and Thandapani considered the equation

$$
\Delta\left(p_{n} \phi\left(\Delta x_{n}\right)\right)+f\left(n, x_{n+1}\right)=0, \quad n=0,1,2, \ldots,
$$

when (1.6) holds and gave some sufficient conditions for the existence of positive solutions.

In this paper we intend to use the Riccati transformation technique for obtaining several new sufficient conditions which guarantee that every solution of equation (1.1) oscillates or converges to zero when (1.6) holds. Our results in this paper are different from those in [1]-[16], [18], [22]-[25]. 


\section{Main results}

TheOREm 2.1. Assume that (1.2) and (1.6) hold. Furthermore, we assume that there exists a positive sequence $\left\{\beta_{n}\right\}_{n=0}^{\infty}$ such that

$\left(h_{1}\right) \Delta \beta_{n} \leq 0, \Delta\left(p_{n} \Delta \beta_{n}\right) \geq 0, \sum_{n=n_{0}}^{\infty} \beta_{n+1} q_{n}=\infty$ and $\sum_{n=n_{0}}^{\infty} \frac{1}{p_{n} \beta_{n}} \sum_{i=n_{0}}^{n-1} \beta_{i+1} q_{i}=\infty$, for some $n_{0}>0$, and

$\left(h_{2}\right) \sum_{i=n+1}^{n+\sigma} Q_{i}>0$ and $\sum_{n=n_{0}}^{\infty} Q_{n}\left[\left(\sum_{i=n+1}^{n+\sigma} Q_{i}\right)^{\frac{1}{1+\sigma}}(\sigma+1)-\sigma\right]=\infty$,

where

$$
Q_{n}=\gamma \frac{n-\sigma}{2\left(p_{n-\sigma}\right)} q_{n}
$$

Then every solution of equation (1.1) oscillates or converges to zero.

Proof. Suppose to the contrary that $\left\{x_{n}\right\}$ is an eventually positive solution of (1.1) such that $x_{n}>0$ and $x_{n-\sigma}>0$ for all $n \geq n_{0}$. We shall consider only this case, since the substitution $y_{n}=-x_{n}$ transforms equation (1.1) into an equation of the same form subject to the assumptions of Theorem. From equation (1.1) we have

$$
\Delta\left(p_{n} \Delta x_{n}\right)=-q_{n} f\left(x_{n-\sigma}\right) \leq 0, \quad n \geq n_{0},
$$

and so $\left\{p_{n} \Delta x_{n}\right\}$ is an eventually nonincreasing sequence. Since $\left\{q_{n}\right\}$ has a positive subsequence, the nondecreasing sequence $\left\{p_{n}\left(\Delta x_{n}\right)\right\}$ is either eventually positive or eventually negative and then from (2.1) there exist two possible cases of $\Delta x_{n}$.

Case (I). Suppose that $\Delta x_{n}<0$ for $n \geq n_{1}>n_{0}$. It follows that $\lim _{n \rightarrow \infty} x_{n}=b \geq 0$. We assert that $\mathrm{b}=0$. If not, then $f\left(x_{n-\sigma}\right) \rightarrow f(b)>$ 0 as $n \rightarrow \infty$. Since $f(x)$ in nondecreasing there exists $n_{2}>n_{1}$ such that $f\left(x_{n-\sigma}\right) \geq f(b)$ for $n \geq n_{2}$. Therefore from (2.1) we have

$$
\Delta\left(p_{n} \Delta x_{n}\right) \leq-q_{n} f(b) .
$$

Define the sequence $u_{n}=\beta_{n}\left(p_{n} \Delta x_{n}\right)$ for $n \geq n_{2}$. Then we have

$$
\Delta u_{n} \leq-f(b) \beta_{n+1} q_{n}+\Delta \beta_{n}\left(p_{n} \Delta x_{n}\right) .
$$

Summing (2.2) from $n_{2}$ to $n-1$, we have

$$
u_{n} \leq u_{n_{2}}-f(b) \sum_{s=n_{2}}^{n-1} \beta_{s+1} q_{s}+\sum_{s=n_{2}}^{n-1}\left(p_{s} \Delta \beta_{s}\right) \Delta x_{s},
$$


and then

$$
u_{n} \leq u_{n_{2}}-f(b) \sum_{s=n_{2}}^{n-1} \beta_{s+1} q_{s}+\left.p_{s} \Delta \beta_{s} \Delta x_{s}\right|_{s=n_{2}} ^{n}-\sum_{s=n_{2}}^{n-1} \Delta\left(p_{s} \Delta \beta_{s}\right) x_{s+1} .
$$

In view of $\left(h_{1}\right)$ we have

$$
u_{n} \leq M-f(b) \sum_{s=n_{2}}^{n-1} \beta_{s+1} q_{s}
$$

where $M=u_{n_{2}}-p_{n_{2}} \Delta \beta_{n_{2}} \Delta x_{n_{2}}$. In view of $\left(h_{1}\right)$, since $\sum_{n=n_{0}}^{\infty} \beta_{n+1} q_{n}=\infty$ it is possible to choose integer $n_{3}$ sufficiently large such that for all $n \geq n_{3}$

$$
u_{n} \leq-\frac{f(b)}{2} \sum_{n=n_{2}}^{n-1} \beta_{s+1} q_{s}
$$

Summing the last inequality from $n_{3}$ to $n$ we obtain

$$
x_{n+1} \leq x_{n_{3}}-\frac{f(b)}{2} \sum_{s=n_{3}}^{n} \frac{1}{p_{s} \beta_{s}} \sum_{i=n_{2}}^{s-1} \beta_{i+1} q_{i} .
$$

Condition $\left(h_{1}\right)$ implies that $\left\{x_{n}\right\}$ is eventually negative, which is a contradiction. Thus $\left\{x_{n}\right\}$ converges to zero.

Case (II). Suppose that $\Delta x_{n}>0$ for $n \geq n_{1}$. Then from equation (1.1) we have $\Delta^{2} x_{n} \leq 0$ for $n \geq n_{1}$, and then $\left\{\Delta x_{n}\right\}$ is nonincreasing sequence, and $x_{n}-x_{n_{1}}=\sum_{k=n_{1}}^{n-1} \Delta x_{k} \geq\left(n-n_{1}\right) \Delta x_{n}$ which implies that $x_{n} \geq \frac{n}{2} \Delta x_{n}$ for $n \geq n_{2} \geq 2 n_{1}+1$. Then

$$
x_{n-\sigma} \geq \frac{n-\sigma}{2} \Delta x_{n-\sigma}, \quad n \geq n_{3}=n_{2}+\sigma .
$$

From equation (1.1) and (1.2) we have

$$
\Delta\left(p_{n} \Delta x_{n}\right)+\gamma q_{n} x_{n-\sigma} \leq 0 .
$$

Then by using (2.4) in (2.5) we have

$$
\Delta\left(p_{n} \Delta x_{n}\right)+\gamma q_{n} \frac{n-\sigma}{2} \Delta x_{n-\sigma} \leq 0, \quad n \geq n_{3},
$$

Setting $y_{n}=p_{n} \Delta x_{n}, y_{n}>0$ and satisfies

$$
\Delta y_{n}+Q_{n} y_{n-\sigma} \leq 0, \quad n \geq n_{3},
$$


where $\mathrm{Q}_{n}=\gamma q_{n} \frac{n-\sigma}{2\left(p_{n-\sigma}\right)}$. Let

$$
\lambda_{n}=-\frac{\Delta y_{n}}{y_{n}} .
$$

Since $\left\{y_{n}\right\}$ is a nonincreasing sequence, then we have $0 \leq \lambda_{n}<1$ for large n. From (2.8) we have $\frac{y_{n+1}}{y_{n}}=1-\lambda_{n}$ and $\frac{y_{n-\sigma}}{y_{n}}=\prod_{i=n-\sigma}^{n-1}\left(1-\lambda_{i}\right)^{-1}$. Then by (2.7) and (2.8) and employing the arithmetic mean-geometric inequality, we have

$$
\lambda_{n} \geq Q_{n} \prod_{i=n-\sigma}^{n-1}\left(1-\lambda_{i}\right)^{-1} \geq Q_{n}\left(1-\frac{1}{\sigma} \sum_{i=n-\sigma}^{n-1} \lambda_{i}\right)^{-\sigma} .
$$

Let $b_{n}=\sum_{i=n+1}^{n+\sigma} Q_{i}$. Then (2.9) can be rewritten as

$$
\lambda_{n} \geq Q_{n}\left(1-\frac{1}{\sigma b_{n}} b_{n} \sum_{i=n-\sigma}^{n-1} \lambda_{i}\right)^{-\sigma} .
$$

Then from (2.10) by using the inequality

$$
\left[1-\frac{1}{\sigma} r x\right]^{-\sigma} \geq x+\frac{\left[r^{\frac{1}{\sigma+1}}(\sigma+1)-\sigma\right]}{r} \text { for } r>0 \text { and } x<\frac{\sigma}{r},
$$

we have

$$
\lambda_{n} \geq Q_{n}\left[\frac{1}{b_{n}} \sum_{i=n-\sigma}^{n-1} \lambda_{i}+\frac{1}{b_{n}}\left(\left(b_{n}\right)^{\frac{1}{\sigma+1}}(\sigma+1)-\sigma\right)\right] .
$$

It follows that

$$
\lambda_{n} b_{n}-Q_{n} \sum_{i=n-\sigma}^{n-1} \lambda_{i} \geq Q_{n}\left(\left(\sum_{i=n+1}^{n+\sigma} Q_{i}\right)^{\frac{1}{\sigma+1}}(\sigma+1)-\sigma\right) .
$$

Then, for $N>n_{3}$,

$$
\sum_{n=n_{3}}^{N} \lambda_{n} b_{n}-\sum_{n=n_{3}}^{N} Q_{n} \sum_{i=n-\sigma}^{n-1} \lambda_{i} \geq \sum_{n=n_{3}}^{N} Q_{n}\left(\left(\sum_{i=n+1}^{n+\sigma} Q_{i}\right)^{\frac{1}{\sigma+1}}(\sigma+1)-\sigma\right) .
$$


Interchanging the bounds of summation, we find

$$
\begin{aligned}
& \sum_{n=n_{3}}^{N} Q_{n} \sum_{i=n-\sigma}^{n-1} \lambda_{i} \geq \sum_{n=n_{3}}^{N-\sigma-1} \sum_{n=i+1}^{i+\sigma} \lambda_{i} Q_{n} \\
= & \sum_{i=n_{3}}^{N-\sigma-1} \lambda_{i} \sum_{n=i+1}^{i+\sigma} Q_{n}=\sum_{n=n_{3}}^{N-\sigma-1} \lambda_{n} \sum_{i=n+1}^{n+\sigma} Q_{i} .
\end{aligned}
$$

Combining (2.13) and (2.14), it follows that

$$
\sum_{n=N-\sigma}^{N} \lambda_{n} \sum_{i=n+1}^{n+\sigma} Q_{i} \geq \sum_{n=n_{3}}^{N} Q_{n}\left(\left(\sum_{i=n+1}^{n+\sigma} Q_{i}\right)^{\frac{1}{\sigma+1}}(\sigma+1)-\sigma\right) .
$$

Summing (2.7) from $n+1$ to $n+\sigma$, we get

$$
y_{n+1+\sigma}-y_{n+1}+\sum_{i=n+1}^{n+\sigma} Q_{i} y_{i-\sigma} \leq 0 .
$$

Using the fact that $\left\{y_{n}\right\}$ is a positive nonincreasing function, we have

$$
y_{n+1}>y_{n} \sum_{i=n+1}^{n+\sigma} Q_{i}
$$

and so

$$
\sum_{i=n+1}^{n+\sigma} Q_{i}<1
$$

eventually. Then, from (2.15) and (2.16) we have

$$
\sum_{n=N-\sigma}^{N} \lambda_{n} \geq \sum_{n=n_{3}}^{N} Q_{n}\left(\left(\sum_{i=n+1}^{n+\sigma} Q_{i}\right)^{\frac{1}{\sigma+1}}(\sigma+1)-\sigma\right) \rightarrow \infty \text { as } N \rightarrow \infty
$$

by $\left(h_{2}\right)$. But, from the definition of $\lambda_{n}$ we have

$$
\lambda_{n}=\left(1-\frac{y_{n+1}}{y_{n}}\right) \text {. }
$$

Hence,

$$
\sum_{n=N-\sigma}^{N} \lambda_{n}=\sum_{n=N-\sigma}^{N}\left(1-\frac{y_{n+1}}{y_{n}}\right)<\sigma+1 .
$$

and this contradicts (2.17). Then every solution of (1.1) oscillates. The proof is complete. 
Note that Theorem 2.1 can not be applied to equation (1.1) when $\sigma=0$. Then the retarded arguments $\sigma$ appearing in the nonlinear term plays an important role in the generating qualitative behavior for equation (1.1) different from that for the corresponding equations with $\sigma=0$. It is of interest to find some new oscillation criteria different from the results in Theorem 2.1.

Theorem 2.2. Assume that (1.2) and (1.6) hold. Furthermore, we assume that there exist positive sequences $\left\{\beta_{n}\right\}_{n=0}^{\infty}$ and $\left\{\rho_{n}\right\}_{n=0}^{\infty}$ such that $\left(h_{1}\right)$ holds, and

$$
\lim _{n \rightarrow \infty} \sup \sum_{l=n_{0}}^{n}\left[\gamma \rho_{l} q_{l}-\frac{p_{l-\sigma}\left(\Delta \rho_{l}\right)^{2}}{4 \rho_{l}}\right]=\infty .
$$

Then every solution of equation (1.1) oscillates or converges to zero.

Proof. We proceed as in Theorem 2.1. We may assume that equation (1.1) has a positive solution $\left\{x_{n}\right\}$ such that $x_{n}>0$ and $x_{n-\sigma}>0$ for all $n \geq n_{0}$. Then we have $\left\{\Delta x_{n}\right\}$ is of one sign. If $\left\{\Delta x_{n}\right\}$ is eventually negative, then we may follow the proof of Theorem 2.1 to show that $\left\{x_{n}\right\}$ converges to zero. Next we consider the second case when $\left\{\Delta x_{n}\right\}$ is positive for all $n \geq n_{1} \geq n_{0}$. From (1.2) and (1.1) we have

$$
\Delta\left(p_{n} \Delta x_{n}\right)+\gamma q_{n} x_{n-\sigma} \leq 0 .
$$

Define the sequence $\left\{w_{n}\right\}$ by

$$
w_{n}=\rho_{n} \frac{p_{n} \Delta x_{n}}{x_{n-\sigma}} .
$$

Then $w_{n}>0$ and

$$
\Delta w_{n}=p_{n+1} \Delta x_{n+1} \Delta\left[\frac{\rho_{n}}{x_{n-\sigma}}\right]+\frac{\rho_{n} \Delta\left(p_{n} \Delta x_{n}\right)}{x_{n-\sigma}}
$$

From (2.21) we have $\Delta\left(p_{n} \Delta x_{n}\right) \leq 0$, and since $\Delta x_{n}>0$, then we conclude that

$$
p_{n-\sigma} \Delta x_{n-\sigma} \geq p_{n+1} \Delta x_{n+1} \text {, and } x_{n+1-\sigma} \geq x_{n-\sigma} \text {. }
$$


From (2.21)-(2.24), we have

$$
\begin{aligned}
\Delta w_{n} & \leq-\gamma \rho_{n} q_{n}+\frac{\Delta \rho_{n}}{\rho_{n+1}} w_{n+1}-\frac{\rho_{n}}{p_{n-\sigma}\left(\rho_{n+1}\right)^{2}} w_{n+1}^{2} \\
& =-\gamma \rho_{n} q_{n}+\frac{p_{n-\sigma}\left(\Delta \rho_{n}\right)^{2}}{4 \rho_{n}}-\left[\frac{\sqrt{\rho_{n} / p_{n-\sigma}}}{\rho_{n+1}} w_{n+1}-\frac{\Delta \rho_{n}}{2 \sqrt{\rho_{n} / p_{n-\sigma}}}\right]^{2} \\
& <-\left[\gamma \rho_{n} q_{n}-\frac{p_{n-\sigma}\left(\Delta \rho_{n}\right)^{2}}{4 \rho_{n}}\right] .
\end{aligned}
$$

Then, we have

$$
\Delta w_{n}<-\left[\gamma \rho_{n} q_{n}-\frac{p_{n-\sigma}\left(\Delta \rho_{n}\right)^{2}}{4 \rho_{n}}\right] .
$$

Summing (2.26) from $n_{1}$ to $n$, we obtain

$$
-w_{n_{1}}<w_{n+1}-w_{n_{1}}<-\sum_{l=n_{1}}^{n}\left[\gamma \rho_{l} q_{l}-\frac{p_{l-\sigma}\left(\Delta \rho_{l}\right)^{2}}{4 \rho_{l}}\right],
$$

which yields

$$
\sum_{l=n_{1}}^{n}\left[\gamma \rho_{l} q_{l}-\frac{p_{l-\sigma}\left(\Delta \rho_{l}\right)^{2}}{4 \rho_{l}}\right] \leq c_{1}
$$

for all large $n$, which is contrary to $(2.20)$. The proof is complete.

From Theorem 2.2, we can obtain different conditions for oscillation of all solutions of equation (1.1) by different choices of $\left\{\rho_{n}\right\}$. Let $\rho_{n}=n^{\lambda}$, $n \geq n_{0}$ and $\lambda \geq 1$ is a constant. By theorem 2.2 we have the following result.

Corollary 2.1. Assume that all the assumptions of Theorem 2.2 hold, except the condition (2.20) is replaced by

$$
\lim _{n \rightarrow \infty} \sup \sum_{s=n_{0}}^{n}\left[\gamma s^{\lambda} q_{s}-\frac{p_{s-\sigma}\left((s+1)^{\lambda}-s^{\lambda}\right)^{2}}{4 s^{\lambda}}\right]=\infty .
$$

Then, every solution of equation (1.1) oscillates or converges to zero.

The following example is illustrative

EXAMPLE 2.1. Consider the difference equation

$$
\Delta\left(n^{2} \Delta x_{n}\right)+\mu x_{n}=0, \quad n \geq 1
$$


where $\mu>\frac{1}{4}$. Then, $p_{n}=n^{2}, \gamma=1$. If we take $\lambda=1$, then we have

$$
\begin{aligned}
& \sum_{s=n_{0}}^{n}\left[\gamma s^{\lambda} q_{s}-\frac{p_{s-\sigma}\left((s+1)^{\lambda}-s^{\lambda}\right)^{2}}{4 s^{\lambda}}\right]=\sum_{s=1}^{n}\left[\mu s-\frac{s^{2}}{4 s}\right] \\
= & \sum_{s=1}^{n} \frac{(4 \mu-1)}{4} s \rightarrow \infty .
\end{aligned}
$$

as $n \rightarrow \infty$. By Corollary 2.1, every solution of (2.30) oscillates or converges to zero. Note that none of the above mentioned papers can be applied to (2.30). Hence, Theorem 2.2 and Corollary 2.1 are sharp.

As a variant of the Riccati transformation technique used above, we will derive a Kamenev type oscillation criteria which can be considered as a discrete analogy of Philos's condition for oscillation of second order differential equations [17].

Theorem 2.3. Assume that (1.2) and (1.5) hold, and let $\left\{\beta_{n}\right\}_{n=0}^{\infty}$ and $\left\{\rho_{n}\right\}_{n=0}^{\infty}$ be two positive sequences such that $\left(h_{1}\right)$ holds. Furthermore, we assume that there exists a double sequence $\left\{H_{m, n}: m \geq n \geq 0\right\}$ such that (i) $H_{m, m}=0$ for $m \geq 0$, (ii) $H_{m, n}>0$ for $m>n>0$, (iii) $\Delta_{2} H_{m, n}=H_{m, n+1}-H_{m, n}$. If

$\lim _{m \rightarrow \infty} \sup \frac{1}{H_{m, 0}} \sum_{n=n_{0}}^{m-1}\left[\gamma H_{m, n} \rho_{n} q_{n}-\frac{\rho_{n+1}^{2}}{4 \bar{\rho}_{n}}\left(h_{m, n}-\frac{\Delta \rho_{n}}{\rho_{n+1}} \sqrt{H_{m, n}}\right)^{2}\right]=\infty$,

where

$$
h_{m, n}=-\frac{\Delta_{2} H_{m, n}}{\sqrt{H_{m, n}}}, \quad \bar{\rho}_{n}=\rho_{n} / p_{n-\sigma} .
$$

Then every solution of equation (1.1) oscillates or converges to zero.

Proof. We proceed as in the proof of Theorem 2.1. We may assume that (1.1) has a nonoscillatory solution $\left\{x_{n}\right\}_{n=0}^{\infty}$. Then we have $\left\{\Delta x_{n}\right\}$ is of one sign. If $\left\{\Delta x_{n}\right\}$ is eventually negative, then we may follow the proof of Theorem 2.1 to show that $\left\{x_{n}\right\}$ converges to zero. Next we consider the case when $\Delta x_{n} \geq 0$ for $n \geq n_{1}$. Define $\left\{w_{n}\right\}$ by $(2.22)$ as before. Then we have $w_{n}>0$ and (2.25) holds. For the sake of convenience, let us set

$$
\bar{\rho}_{n}=\rho_{n} / p_{n-\sigma} .
$$

Then,

$$
\gamma \rho_{n} q_{n} \leq-\Delta w_{n}+\frac{\Delta \rho_{n}}{\rho_{n+1}} w_{n+1}-\frac{\bar{\rho}_{n}}{\left(\rho_{n+1}\right)^{2}} w_{n+1}^{2}
$$


Therefore, we have

$$
\begin{aligned}
& \sum_{n=n_{1}}^{m-1} \gamma H_{m, n} \rho_{n} q_{n} \\
\leq & -\sum_{n=n_{1}}^{m-1} H_{m, n} \Delta w_{n}+\sum_{n=n_{1}}^{m-1} H_{m, n} \frac{\Delta \rho_{n}}{\rho_{n+1}} w_{n+1}-\sum_{n=n_{1}}^{m-1} H_{m, n} \frac{\bar{\rho}_{n}}{\left(\rho_{n+1}\right)^{2}} w_{n+1}^{2}
\end{aligned}
$$

which yields, after summing by parts

$$
\begin{aligned}
& \sum_{n=n_{1}}^{m-1} \gamma H_{m, n} \rho_{n} q_{n} \\
\leq & H_{m, n_{1}} w_{n_{1}}+\sum_{n=n_{1}}^{m-1} w_{n+1} \Delta_{2} H_{m, n}+\sum_{n=n_{1}}^{m-1} H_{m, n} \frac{\Delta \rho_{n}}{\rho_{n+1}} w_{n+1} \\
& -\sum_{n=n_{1}}^{m-1} H_{m, n} \frac{\bar{\rho}_{n}}{\left(\rho_{n+1}\right)^{2}} w_{n+1}^{2} \\
= & H_{m, n_{1}} w_{n_{1}}-\sum_{n=n_{1}}^{m-1} h_{m, n} \sqrt{H_{m, n}} w_{n+1}+\sum_{n=n_{1}}^{m-1} H_{m, n} \frac{\Delta \rho_{n}}{\rho_{n+1}} w_{n+1} \\
& -\sum_{n=n_{1}}^{m-1} H_{m, n} \frac{\bar{\rho}_{n}}{\left(\rho_{n+1}\right)^{2}} w_{n+1}^{2}=H_{m, n_{1}} w_{n_{1}} \\
& -\sum_{n=n_{1}}^{m-1}\left[\frac{\sqrt{H_{m, n} \bar{\rho}_{n}}}{\rho_{n+1}} w_{n+1}\right. \\
& +\frac{1}{4} \sum_{n=n_{1}}^{m-1} \frac{\left(\rho_{n+1}\right)^{2}}{\bar{\rho}_{n}}\left(h_{m, n}-\frac{\Delta \rho_{n}}{\rho_{n+1}} \sqrt{H_{m, n}}\right)^{2}
\end{aligned}
$$

Then,

$$
\begin{aligned}
& \sum_{n=n_{1}}^{m-1}\left[\gamma H_{m, n} \rho_{n} q_{n}-\frac{\left(\rho_{n+1}\right)^{2}}{4 \rho_{n}}\left(h_{m, n}-\frac{\Delta \rho_{n}}{\rho_{n+1}} \sqrt{H_{m, n}}\right)^{2}\right]<H_{m, n_{1}} w_{n_{1}} \\
\leq & H_{m, 0} w_{n_{1}}
\end{aligned}
$$


which implies that

$$
\begin{aligned}
& \sum_{n=0}^{m-1}\left[\gamma H_{m, n} \rho_{n} q_{n}-\frac{\left(\rho_{n+1}\right)^{2}}{4 \bar{\rho}_{n}}\left(h_{m, n}-\frac{\Delta \rho_{n}}{\rho_{n+1}} \sqrt{H_{m, n}}\right)^{2}\right] \\
< & H_{m, 0}\left(w_{n_{1}}+\sum_{n=0}^{n_{1}-1} \gamma \rho_{n} q_{n}\right) .
\end{aligned}
$$

Hence

$$
\begin{aligned}
& \lim _{m \rightarrow \infty} \sup \frac{1}{H_{m, 0}} \sum_{n=0}^{m-1}\left[\gamma H_{m, n} \rho_{n} q_{n}-\frac{\left(\rho_{n+1}\right)^{2}}{4 \bar{\rho}_{n}}\left(h_{m, n}-\frac{\Delta \rho_{n}}{\rho_{n+1}} \sqrt{H_{m, n}}\right)^{2}\right] \\
< & \left(w_{n_{1}}+\sum_{n=0}^{n_{1}-1} \gamma \rho_{n} q_{n}\right)<\infty,
\end{aligned}
$$

which is contrary to (2.31). The proof is complete.

By choosing the sequence $\left\{H_{m, n}\right\}$ in appropriate manners, we can derive several oscillation criteria for (1.1). For instance, let us consider the double sequence $\left\{H_{m, n}\right\}$ defined by

$$
\left.\begin{array}{ll}
H_{m, n}=(m-n)^{\lambda}, & \lambda \geq 1, m \geq n \geq 0, \\
H_{m, n}=\left(\log \frac{m+1}{n+1}\right)^{\lambda}, & \lambda \geq 1, m \geq n \geq 0, \\
H_{m, n}=(m-n)^{(\lambda)} & \lambda>2, m \geq n \geq 0,
\end{array}\right\}
$$

where $(m-n)^{(\lambda)}=(m-n)(m-n+1) \cdots(m-n+\lambda-1)$, and

$$
\Delta_{2}(m-n)^{(\lambda)}=(m-n-1)^{(\lambda)}-(m-n)^{(\lambda)}=-\lambda(m-n)^{(\lambda-1)} .
$$

Then $H_{m, m}=0$ for $m \geq 0$ and $H_{m, n}>0$ and $\Delta_{2} H_{m, n} \leq 0$ for $m>$ $n>\geq 0$. Hence we have the following results.

Corollary 2.2. Assume that all the assumptions of Theorem 2.3 hold, except the condition (2.31) is replaced by

$$
\begin{aligned}
\lim _{m \rightarrow \infty} & \sup \frac{1}{m^{\lambda}} \sum_{n=0}^{m-1}\left[\gamma(m-n)^{\lambda} \rho_{n} q_{n}\right. \\
& \left.-\frac{p_{n-\sigma} \rho_{n+1}^{2}}{4 \rho_{n}}\left(\lambda(m-n)^{\frac{\lambda-2}{2}}-\frac{\Delta \rho_{n}}{\rho_{n+1}} \sqrt{(m-n)^{\lambda}}\right)^{2}\right]=\infty .
\end{aligned}
$$

Then every solution of equation (1.1) oscillates or converges to zero. 
Corollary 2.3. Assume that all the assumptions of Theorem 2.3 hold, except the condition (2.31) is replaced by (2.35)

$$
\begin{aligned}
& \lim _{m \rightarrow \infty} \sup \frac{1}{(\log (m+1))^{\lambda}} \sum_{n=0}^{m-1}\left[\gamma\left(\log \frac{m+1}{n+1}\right)^{\lambda} \rho_{n} q_{n}\right. \\
& \left.-\frac{p_{n-\sigma} \rho_{n+1}^{2}}{4 \rho_{n}}\left(\frac{\lambda}{n+1}\left(\log \frac{m+1}{n+1}\right)^{\frac{\lambda-2}{2}}-\frac{\Delta \rho_{n}}{\rho_{n+1}} \sqrt{\left(\log \frac{m+1}{n+1}\right)^{\lambda}}\right)^{2}\right] \\
& =\infty
\end{aligned}
$$

Then, every solution of equation (1.1) oscillates or converges to zero.

Corollary 2.4. Assume that all the assumptions of Theorem 2.3 hold, except the condition (2.31) is replaced by

$$
\begin{aligned}
\lim _{m \rightarrow \infty} & \sup \frac{1}{m^{(\lambda)}} \sum_{n=0}^{m-1}(m-n)^{(\lambda)}\left[\gamma \rho_{n} q_{n}\right. \\
& \left.-\frac{p_{n-\sigma} \rho_{n+1}^{2}}{4 \rho_{n}}\left(\frac{\lambda}{m-n+\lambda-1}-\frac{\Delta \rho_{n}}{\rho_{n+1}}\right)^{2}\right]=\infty .
\end{aligned}
$$

Then, every solution of equation (1.1) oscillates or converges to zero.

ACKNOWLEDGEMENT. The author thanks the referee for helpful suggestions to improve the language of the paper.

\section{References}

[1] R. P. Agarwal, Difference Equations and Inequalities, Theory, methods and applications, Second edition, Marcel Dekker, New York, 2000.

[2] R. P. Agarwal and P. J. Y. Wong, Advanced topics in difference equations, Kluwer Academic Publishers, Dordrecht, 1997.

[3] M. H. Abu-Risha, Oscillation of second-order linear difference equation, Appl. Math. Lett. 13 (2000), 129-135.

[4] R. Arul and E. Thandapani, Asymptotic behavior of positive solutions of second order quasilinear difference equations, Kyungpook Math. J. 40 (2000), 275-286.

[5] S. S. Cheng, and H. J. Li, A comparison theorems for asymptotically monotone solutions of nonlinear difference equations, Bull. Inst. Math. Acad. Sinica 21 (1993), 299-302.

[6] _ـ Bounded solutions of nonlinear difference equations, Tamkang J. Math. 21 (1990), 137-142.

[7] S. S. Cheng, T. C. Yan and H. J. Li, Oscillation criteria for second order difference equation, Funkcial. Ekvac. 34 (1991), 223-239. 
[8] S. C. Fu and L. Y. Tsai, Oscillation in nonlinear difference equations, Comput. Math. Appl. 36 (1998), 193-201.

[9] S. R. Grace and H. A. El-Morshedy, Oscillation and nonoscillation theorems for certain second-order difference equations with forcing term, J. Math. Anal. Appl. 216 (1997), 614-625.

[10] X. Z. He, Oscillatory and asymptotic behavior of second order nonlinear difference equations, J. Math. Anal. Appl. 175 (1993), 482-498.

[11] H. J. Li and S. S. Cheng, An oscillation theorem for a second order nonlinear difference equation, Utilitas Mathematica 44 (1993), 177-181.

[12] _ Asymptotically monotone solutions of a nonlinear difference equation, Tamkang J. Math. 24 (1993), 269-282.

[13] W. T. Li, Oscillation theorems for second order nonlinear difference equations, Math. Comput. Modelling 31 (2000), 71-79.

[14] W. T. Li and S. S. Cheng, Oscillation criteria for a nonlinear difference equation, Comp. Math. Appl. 36 (1998), 87-94.

[15] W. T. Li, X. L. Fan, C. K. Zhong, On unbounded positive solutions of secondorder difference equations with a singular nonlinear term, J. Math. Anal. Appl. 246 (2000), 80-88.

[16] Z. R. Liu, W. D. Chen and Y. H. Yu, Oscillation criteria for second order nonlinear delay difference equations, Kyungpook Math. J. 39 (1999), 127-132.

[17] Ch. G. Philos, Oscillation theorems for linear differential equations of second order, Arch. Math. 53 (1989), 482-492.

[18] P. Rehak, Hartman-Wintner type lemma, oscillation and conjugacy criteria for half-linear difference equations, J. Math. Anal. Appl. 252 (2000), 813-827.

[19] S. H. Saker, Kamenev-type oscillation criteria for forced Emden-Fowler superlinear difference equations, Electron. J. Differential Equations 2002 (2002), no. $68,1-9$.

[20] _ New oscillation criteria for second-order nonlinear neutral delay difference equations, Applied Math. Comput. 142 (2003), 99-111.

[21] $\_$, Oscillation of second-order perturbed nonlinear difference equations, Appl. Math. Comput. (in press).

[22] B. Szmanda, Characterization of oscillation of second order nonlinear difference equations, Bull. Polish Acad. Sci. Math. 34 (1986), 133-141.

[23] E. Thandapani and K. Ravi, Oscillation of second-order half-linear difference equations, Appl. Math. Lett. 13 (2000), 43-49.

[24] B. G. Zhang, Oscillation and asymptotic behavior of second order difference equations, J. Math. Anal. Appl. 173 (1993), 58-68.

[25] Z. Zhang and J. Zhang, Oscillation criteria for second-order functional difference equations with "summation small" coefficients, Comput. Math. Appl. 38 (1999), $25-31$.

Mathematics Department, Faculty of Science, Mansoura University, ManSOURA, 35516, EGYPT

E-mail: shsaker@mum.mans.eun.eg 